AIDS Behav. 2018 January ; 22(1): 1-8. doi:10.1007/s10461-017-1906-3.

\title{
Systematic Review of Interventions for Depression for People Living with HIV in Africa
}

\author{
Sarah Lofgren ${ }^{1,2}$, Noeline Nakasujja ${ }^{2,3}$, and David R Boulware, MD ${ }^{1}$ \\ ${ }^{1}$ University of Minnesota Department of Medicine, Division of Infectious Diseases and \\ International Medicine, 420 Delaware Stree, Minneapolis, MN 55455, USA 2 Infectious Disease \\ Institute, P.O.Box 22418, Kampala, Kampala, Uganda ${ }^{3}$ Makerere University, Department of \\ Psychiatry, Makerere University College of Health Sciences, Mulago Hill Complex, Kampala, \\ Uganda
}

\begin{abstract}
Interventions for depression among individuals with HIV/AIDS in Africa are being increasingly evaluated. Using key terms: depression, Africa, and HIV, we searched MEDLINE to identify depression interventions for adults with HIV in Africa. Women in the perinatal period were excluded. Results were extracted and weighted relative change in depression scores for interventions and net effect calculated. Search yielded 18 articles. Six of seven studies evaluating feasibility were positive versus seven positive studies evaluating acceptability. Three studies investigated psychotherapy (73\% intervention: $39 \%$ net decrease). Four studies investigated taskshifting of psychotherapy (47\% intervention: $34 \%$ net decrease). Three studies evaluated antidepressants (79\% intervention: 39\% net decrease). Three studies investigated task-shifting of antidepressant treatment (82\% intervention: $65 \%$ net decrease). An exercise intervention was evaluated (66\% intervention: $49 \%$ net decrease). One trial investigated minocycline with insignificant results. Finally, three studies investigated other psychosocial interventions (44\% intervention: $21 \%$ net decrease). Results highlight the need for large, randomized efficacy as well as implementation studies.
\end{abstract}

\section{Keywords \\ HIV; Depression; Africa; Intervention}

\section{Background}

Depression is known to be a major cause of morbidity and mortality with an estimated loss of 63 million disability-adjusted life years (DALY) compared with 82 million for HIV. ${ }^{1}$ Depression is also known to cause 2.2 million excess deaths globally in 2010 from suicide and comorbid diseases. ${ }^{2}$ Depression is significantly more common in HIV infected persons where the global prevalence is $12.8-78 \%$ compared with $4.7 \%$ prevalence in the general

Corresponding Author: Sarah Lofgren, Division of Infectious Disease and International Medicine, 420 Delaware Street, SE, MMC 250, Minneapolis, MN 55455, Lofg0020@umn.edu, (612) 626-9943, Fax: (612) 625-4410. 
population. ${ }^{3,4}$ This is especially concerning as people living with HIV and depression are $42 \%$ less likely to achieve good antiretroviral therapy (ART) adherence, more likely to develop virologic failure, and are more likely to have HIV progression, independent of ART adherence, than those without depression. ${ }^{3,5,6}$

In Africa, which has the highest burden of HIV depression treatment is especially difficult. The paucity of resources, infrastructure, and staff are significant impediments to depression care. In Africa, there are 0.5 psychiatrists per million people compared to 83 per million in Europe 37 per million in South America, 3 per million in India, 124 per million in the USA, and 134 per million in Canada. ${ }^{7}$ This also varies across Africa with 7.8 psychiatrists per two million in South Africa versus zero in Eritrea. ${ }^{7}$ In spite of difficulties there has been significant research in Africa into the prevalence and incidence of comorbid HIV and depression, confounding factors such as alcohol abuse and trauma. There also has been much work on the validation of depression screening tools. While there have been review articles investigating interventions for mental illness in people living with HIV/AIDS in low and middle income countries these have not been specific to depression interventions in Africa.

The purpose of this systematic review is to evaluate interventions for depression care in Africa, including the use of medications, psychotherapy, or other methods.

\section{Methods}

\section{Systematic Search}

MEDLINE was searched using the terms HIV and Africa and Depression. This resulted in the following MeSH terms being used "HIV," "depressive disorder," "depression," and "Africa." All Fields were searched for "HIV," "depressive," "disorder," "depressive disorder," "depression," and "Africa." This returned 545 articles.

Selected articles had to meet the following criteria: 1) have depression as an objective, measure depression using a validated tool and show outcome results, 2) target adults living with HIV, 3) be conducted in Africa, 4) be an intervention other than antiretroviral medication, and 5) focus on depression treatment, not just screening. Article exclusion criteria included 1) peri-partum depression and 2) pediatric studies. These were excluded as there was a recent excellent review on peri-partum depression, ${ }^{14}$ and as the interventions for children, especially young children, are distinct from adults. A wide range of rigor was allowed in this systematic review in order to summarize the available data. Thus, we included those with or without a control arm, feasibility studies, and pilot studies.

\section{Data Analysis}

From each selected article, data were extracted regarding study population, country, year, and study design. Additionally, we assessed if depression was a primary or secondary objective, the depression scale(s) utilized, sample size, inclusion criteria, and outcome data. Descriptive comparisons were performed using Microsoft Excel. Feasibility and acceptability were defined by the individual studies. 
The studies were grouped by intervention type and compared by relative change in depression score. Since the studies used different depression scores to measure the depression, we compared the result by relative change in the depression score. The effect score was divided by the baseline score. Since this fraction gave the remaining depression and not the reduction in depression, the fraction was subtracted from one and the result multiplied by $100 \%$. Control reduction was calculated in the same way. If there was a control arm a net reduction of depression score was calculated by subtracting the control reduction from the intervention reduction. This methodology was also chosen as several studies gave the mean depression scores at baseline and after the intervention but not the number or percentage of those who went from depressed to not depressed defined as the response rate.

\section{Results}

We completed our search on February 3, 2017 and did not limit articles by date. Our search yielded 545 articles. The article titles were screened for inclusion/exclusion, leaving 50 articles. The abstracts were reviewed and 24 were removed. The full texts of the remaining 26 were reviewed and eight more were removed. Full search details can be seen in Figure 1.

The 18 included articles were read and data abstracted. Their main points are summarized in Table 1. Although the search was not limited to Sub-Saharan Africa, all of the studies were from there. Most of the articles described pilot studies. The type of interventions and depression scales are varied.

Several trials investigated the feasibility and/or acceptability of the intervention. The feasibility was generally defined as by the ability to recruit staff and participants, retention of participants, the ability to collect desired data, and the fidelity of completing the intervention as defined. Seven studies accessed feasibility of their methods, six assessing psychotherapy as well as the three looking at task shifting of antidepressant assessment and delivery. Of the seven, only one, Andersen, et al., expressed concerns about feasibility given the difficulty in fidelity of the intervention, ${ }^{8}$ all of the other six studies found that providing the intervention was feasible. ${ }^{9-14}$ Seven studies evaluated acceptability and all found the respective intervention acceptable. The acceptability was defined as either as patient retention and adherence to the intervention or through qualitative interviews or surveys. The seven studies included psychotherapy $(n=5)^{8-11,14}$ and task shifting of antidepressant delivery $(n=2) .{ }^{12,13}$ Thus, the feasibility and acceptability was high.

To evaluate the validity of our comparison of results between studies, we examined two studies which used two different depression scores. Andersen, et al. used both Center for Epidemiological Studies Depression Scale (CES-D) and the Hamilton Depression Scale (HAM-D) which yielded percent reduction of intervention scores of $16 \%$ and $22 \%$ respectively. ${ }^{11}$ Hoare, et al. used both the HAM-D and the Montgomery-Asberg depression rating scale ${ }^{12}$ (MADRS) which yielded percent reduction of intervention scores of $60 \%$ and $56 \% .{ }^{15}$ Thus, the percent reduction appeared to generally result in approximately similar relative responses across different metrics. 


\section{Psychotherapy}

Overall in the psychotherapy trials, there was significant reduction in depression as summarized in Table 2a. ${ }^{16-18}$ Overall weighted reduction in the score of the intervention group was $73 \%$ and the weighted net reduction was $39 \%$ when removing the effect observed in the control group. The authors did not report the percent response; however, the means for each group are below the cutoff for even borderline depression. Of note the participants in Nakimuli-Mpungu, et al. were allowed to utilize antidepressants as needed. ${ }^{16}$

\section{Cognitive Behavioral Therapy Task-shifted Intervention}

Also in the task shifted psychotherapy groups there was significant reduction in depression as summarized in Table $2 \mathrm{~b} .{ }^{8-11}$ Overall weighted reduction of the score in the intervention group was $47 \%$. Only two of the task-shifted psychotherapy studies included controls. The weighted average net reduction was $34 \%$ in those studies. These studies also did not report response rates, however again the average score in each study was below the cutoff for depression. In Abas, et al the participants were allowed to take antidepressants as needed. Those participants were also on ART but poorly adherent and adherence counseling was part of the intervention. Anderson, et al also allowed participants to take antidepressants but the doses could not have been adjusted in the 3 months prior to enrolling in the study. Those participants were also on ART and two thirds were maintaining > 80\% adherence.

\section{Antidepressants trials}

There were three antidepressants trials, two reported positive response, ${ }^{19,20}$ while one reported no significant results as summarized in Table $2 \mathrm{c} .{ }^{15}$ The average weighted reduction of intervention score was $79 \%$. Only the negative study had a control with the net reduction of $0 \% .{ }^{15}$ These studies did give response rates. The negative study showed only $3 \%$ of their participants going from depressed to not depressed; however, the other two reported a 79\% and $86 \%$ result. The overall weighted rate was $61 \%$ response rate. All participants were drawn from HIV clinics. Rates of ART use was only described in one of study, the Wagner, et al. cohorts, in which all participants were about to start ART at enrollment. ${ }^{20}$

\section{Antidepressant Task-Shifted Studies}

There were three studies examining task shifting in the screening for and administration of antidepressants as summarized in Table $2 \mathrm{~d} .{ }^{12,21,22}$ Since these studies were pilot studies looking at feasibility none of them have controls. Each reported positive responses and all showed feasibility. The weighted average reduction of intervention score was $82 \%$. Two studies reported response rates for weighted average of $65 \%$. The third study's mean follow up score was below the threshold for depression. Participants from Adams, et al. were drawn from the HIV clinic although ART history was not provided. Participants from Gaynes, et al. were on ART although poorly adherent with $53 \%$ reporting $95 \%$ or more adherence at baseline and $63 \%$ at 4 months. They also had $0 \%$ virologically suppressed at baseline and $18 \%$ at 4 months. In Pence et al. the participants were drawn from ART clinic but none were virologically suppressed at baseline. Only one participant had ever been treated for depression before although $96 \%$ reported at least one prior depressive episode. 


\section{Exercise Study}

A novel study used exercise to improve pulmonary function as well as mental health as summarized in Table $2 \mathrm{e}^{23}$ They had $66 \%$ reduction in the depression symptoms and a net reduction of $49 \%$. There was no response rate reported. However, the mean final score is below the cutoff for depression. The participants were all on ART but adherence information was not given. It was not noted if they could be on antidepressants or not.

\section{Novel Drug}

In 2013 a trial in Uganda examined minocycline utilization for cognitive function. The authors measured depression as well; their results are summarized in Table 2f. ${ }^{24}$ The participants were allowed to be on antidepressants as needed. Unfortunately, the effect of minocycline was not significant for cognitive function or depression and the trial was stopped early.

\section{Other Psychosocial Intervention}

Finally, there were three studies analyzing other psychosocial interventions that evaluated depression as summarized in Table $2 \mathrm{~g} .{ }^{25-27}$ These included two stigma reduction interventions of people living with HIV and those around them and a community accompaniment intervention using community health workers. Their response rates were more modest; however not all participants started with depression and depression was a secondary endpoint. The weighted reduction in the intervention score was $39 \%$ and net reduction in the intervention result was $21 \%$. The intervention arm from the community accompaniment trial had $44.3 \%$ greater response than the other arm. Information regarding ART use was not noted in Chidrawi, et al. or Prinsloo, et al. although both studies were drawn from people in care. Those in Thomson, et al. were initiating ART. None of the studies mentioned antidepressants.

The results of this analysis are shown in Figure 2. Note that while all three of the psychotherapy studies included control arms, only two of those with task shifted psychotherapy did. Most notably, in the antidepressant studies only one had a control arm and overall there was no net change in that study. Those in the task shifted antidepressant trials did not have control arms and only one in the other psychosocial interventions had a control arm.

\section{Discussion}

This review demonstrates that a variety of depression treatment modalities have been tried in Africa. These trials are diverse and were completed across the continent which suggests wide applicability. While there were challenges, such as pharmacy outages in drug delivery, a high percentage of patients experienced favorable results. While it is difficult to compare between studies and across modalities, both psychotherapy and antidepressants seem to have efficacy in low-resource areas, the same as in high-resource areas.

As mentioned above, since we were comparing depression scores from different scales we did relative reductions in score. To evaluate the validity of this approach we looked at two 
studies that used more than one depression score. Andersen, et al. used both Center for Epidemiological Studies Depression Scale (CES-D) and the Hamilton Depression Scale (HAM-D) which yielded percent reduction of intervention scores of $16.1 \%$ and $22 \%$ respectively. Hoare, et al. used both the Montgomery-Asberg depression rating scale (MADRS) and the HAM-D which yielded percent reduction of intervention scores of 56.2\% and $60.2 \%$. We felt that these differences were acceptable and added support to our procedure for comparison of relative depression scores.

All of the psychotherapy studies reported at least a 40 percent decrease in initial depression score and one study reported a reduction of over eighty percent. All of those with control arms showed a reduction over the control reduction as well. The studies were small and underpowered to show efficacy. Thus while we think it is reasonable to summarize the data, this review is not purporting that the efficacy is proved but promising In spite of that the results are promising given the high acceptability and feasibility.

Five of the antidepressant trials showed reduction in depression score of at least 59\%. There was one negative trial; however, they only allowed a fixed dose of escitalopram 10mg and no titration up in spite of $20 \mathrm{mg}$ being a safe dose. Seeing a trial that could titrate the escitalopram dose would provide more information. However even including the results of that trial, the response in Africa is quite positive.

Aweto, et al showed that patients with HIV had significant benefit from exercise. These findings are encouraging given the low cost and wide applicability of such an intervention. Given the rising rates of obesity in the developing world, exercise interventions for depression would likely have other beneficial side effects.

While the trials looking at other psychosocial interventions did not have as much reduction in depression score as some of the other interventions, they also did not choose only depressed patients. Thus it is somewhat impressive they saw a treatment benefit at all. The community accompaniment study had significant reductions in rates of depression. Subanalysis using only people testing as depressed initially, or developing a new trial analyzing stigma would be valuable to determine benefit from intervention within these groups of people.

Finally, nearly all of these studies were pilot studies, many with small sample sizes.

However, it seems possible to conclude that depression treatment is feasible, acceptable and worthwhile in resource-limited settings in Africa. Given low personnel costs in Africa as well as the ability to task-shift, psychotherapy would likely not be resource intensive. Antidepressants seem to have promise in Africa, likely limited by pharmacy supply. Novel approaches such as exercise, sigma reduction, or community accompaniment when starting ART appear to have merit. There is clearly a need for larger trials to be more certain of efficacy and response rates. However, given the known poorer HIV outcomes of those with depression, ${ }^{3,5}$ treating depression seems a necessary target to try to achieve the UNAIDs 90-90-90 goals in people living with HIV in Africa. 


\section{Conclusion}

Depression is an important modulating factor in HIV outcomes in Africa. In spite of that, research into depression interventions have largely only come recently. These interventions show that there are many impactful ways to improve the lives of those with HIV in Africa, and in turn improve HIV outcomes. While larger studies are needed to show efficacy, there is clear evidence that psychotherapy and antidepressant delivery are feasible and acceptable in Africa. Also there are some promising alternatives such as exercise or community accompaniment strategies which would not just impact depression but overall physical or psychosocial health. Work into implementation of these strategies are needed to put interventions into practice as part of holistic HIV care in Africa.

\section{Limitations}

Many of these trials were feasibility or pilot studies so were not powered for response. Negative response would not necessarily mean no response. The fact that there were significant responses in many of these articles is all the more impressive given their power. There were many depression scales used and in an effort to compare across scales we used relative change. This is useful but not perfect and would certainly not imply high precision of measurement. Greater agreement in depression scales in Africa would help compare depression rates and efficacy. Finally MEDLINE was the only database searched. However, all the references found were searched to look for articles cited by the authors to looked for missed articles. No missed articles were found.

\section{Acknowledgments}

This research was supported by the National Institute of Neurologic Diseases and Stroke (NINDS) and Fogarty International Center (R01NS086312, R25TW009345) and NIAID (T32AI055433).

\section{References}

1. Murray CJ, Vos T, Lozano R, et al. Disability-adjusted life years (DALYs) for 291 diseases and injuries in 21 regions, 1990-2010: a systematic analysis for the Global Burden of Disease Study 2010. Lancet. 2012; 380(9859):2197-2223. [PubMed: 23245608]

2. Patel V, Chisholm D, Parikh R, et al. Addressing the burden of mental, neurological, and substance use disorders: key messages from Disease Control Priorities, 3rd edition. Lancet. 2016; 387(10028): 1672-1685. [PubMed: 26454360]

3. Uthman OA, Magidson JF, Safren SA, Nachega JB. Depression and adherence to antiretroviral therapy in low-, middle- and high-income countries: a systematic review and meta-analysis. Curr HIV/AIDS Rep. 2014; 11(3):291-307. [PubMed: 25038748]

4. Ferrari AJ, Somerville AJ, Baxter AJ, et al. Global variation in the prevalence and incidence of major depressive disorder: a systematic review of the epidemiological literature. Psychol Med. 2013; 43(3):471-481. [PubMed: 22831756]

5. Bouhnik AD, Preau M, Vincent E, et al. Depression and clinical progression in HIV-infected drug users treated with highly active antiretroviral therapy. Antivir Ther. 2005; 10(1):53-61. [PubMed: 15751763]

6. Marconi VC, Wu B, Hampton J, et al. Early warning indicators for first-line virologic failure independent of adherence measures in a South African urban clinic. AIDS Patient Care STDS. 2013; 27(12):657-668. [PubMed: 24320011]

7. World Health Organization. [Accessed April 15, 2017] Mental Health. Global Health Observatory Data. 2017. http://www.who.int/gho/mental_health/en/ 2017 
8. Andersen LS, Magidson JF, O'Cleirigh C, et al. A pilot study of a nurse-delivered cognitive behavioral therapy intervention (Ziphamandla) for adherence and depression in HIV in South Africa. J Health Psychol. 2016

9. Abas M, Nyamayaro P, Bere T, et al. Feasibility and Acceptability of a Task-Shifted Intervention to Enhance Adherence to HIV Medication and Improve Depression in People Living with HIV in Zimbabwe, a Low Income Country in Sub-Saharan Africa. AIDS Behav. 2017

10. Chibanda D, Mesu P, Kajawu L, Cowan F, Araya R, Abas MA. Problem-solving therapy for depression and common mental disorders in Zimbabwe: piloting a task-shifting primary mental health care intervention in a population with a high prevalence of people living with HIV. BMC Public Health. 2011; 11:828. [PubMed: 22029430]

11. Petersen I, Hanass Hancock J, Bhana A, Govender K. A group-based counselling intervention for depression comorbid with HIV/AIDS using a task shifting approach in South Africa: a randomized controlled pilot study. J Affect Disord. 2014; 158:78-84. [PubMed: 24655769]

12. Adams JL, Almond ML, Ringo EJ, Shangali WH, Sikkema KJ. Feasibility of nurse-led antidepressant medication management of depression in an HIV clinic in Tanzania. Int J Psychiatry Med. 2012; 43(2):105-117. [PubMed: 22849034]

13. Pence BW, Gaynes BN, Atashili J, et al. Feasibility, safety, acceptability, and preliminary efficacy of measurement-based care depression treatment for HIV patients in Bamenda, Cameroon. AIDS Behav. 2014; 18(6):1142-1151. [PubMed: 24558099]

14. Nakimuli-Mpungu EWK, Okello J, Alderman S, Odokonyero R, Musisi S, Mojtabai R, Mills E. Outcomes, feasibility and acceptability of a group support psychotherapeutic intervention for depressed HIV affected Adults: A pilot study. Journal of Affective Disorders. 2014; 166:144-150. [PubMed: 25012423]

15. Hoare J, Carey P, Joska JA, Carrara H, Sorsdahl K, Stein DJ. Escitalopram treatment of depression in human immunodeficiency virus/acquired immunodeficiency syndrome: a randomized, doubleblind, placebo-controlled study. J Nerv Ment Dis. 2014; 202(2):133-137. [PubMed: 24469525]

16. Nakimuli-Mpungu E, Wamala K, Okello J, et al. Outcomes, feasibility and acceptability of a group support psychotherapeutic intervention for depressed HIV-affected Ugandan adults: a pilot study. J Affect Disord. 2014; 166:144-150. [PubMed: 25012423]

17. Nakimuli-Mpungu E, Wamala K, Okello J, et al. Group support psychotherapy for depression treatment in people with HIV/AIDS in northern Uganda: a single-centre randomised controlled trial. Lancet HIV. 2015; 2(5):e190-199. [PubMed: 26423001]

18. Olley BO. Improving well-being through psycho-education among voluntary counseling and testing seekers in Nigeria: a controlled outcome study. AIDS Care. 2006; 18(8):1025-1031. [PubMed: 17012095]

19. Ngo VK, Wagner GJ, Nakasujja N, Dickens A, Aunon F, Musisi S. Effectiveness of antidepressants and predictors of treatment response for depressed HIV patients in Uganda. Int J STD AIDS. 2015; 26(14):998-1006. [PubMed: 25525053]

20. Wagner GJ, Ngo VK, Nakasujja N, Akena D, Aunon F, Musisi S. Impact of antidepressant therapy on cognitive aspects of work, condom use, and psychosocial well-being among HIV clients in Uganda. Int J Psychiatry Med. 2014; 48(3):155-166. [PubMed: 25492710]

21. Gaynes BN, Pence BW, Atashili J, et al. Changes in HIV Outcomes Following Depression Care in a Resource-Limited Setting: Results from a Pilot Study in Bamenda, Cameroon. PLoS One. 2015; 10(10):e0140001. [PubMed: 26469186]

22. Belenky NM, Cole SR, Pence BW, Itemba D, Maro V, Whetten K. Depressive symptoms, HIV medication adherence, and HIV clinical outcomes in Tanzania: a prospective, observational study. PLoS One. 2014; 9(5):e95469. [PubMed: 24798428]

23. Aweto HA, Aiyegbusi AI, Ugonabo AJ, Adeyemo TA. Effects of Aerobic Exercise on the Pulmonary Functions, Respiratory Symptoms and Psychological Status of People Living With HIV. J Res Health Sci. 2016; 16(1):17-21. [PubMed: 27061991]

24. Nakasujja N, Miyahara S, Evans S, et al. Randomized trial of minocycline in the treatment of HIVassociated cognitive impairment. Neurology. 2013; 80(2):196-202. [PubMed: 23269596]

25. Chidrawi HC, Greeff M, Temane QM, Ellis S. Changeover-time in psychosocial wellbeing of people living with HIV and people living close to them after an HIV stigma reduction and 
wellness enhancement community intervention. Afr J AIDS Res. 2015; 14(1):1-12. [PubMed: 25920979]

26. Prinsloo CD, Greeff M. A Community "Hub" Network Intervention for HIV Stigma Reduction: A Case Study. J Assoc Nurses AIDS Care. 2016; 27(2):166-179. [PubMed: 26627447]

27. Thomson DR, Rich ML, Kaigamba F, et al. Community-based accompaniment and psychosocial health outcomes in HIV-infected adults in Rwanda: a prospective study. AIDS Behav. 2014; 18(2): 368-380. [PubMed: 23443977] 


\section{Search February 2017} Medline: 545

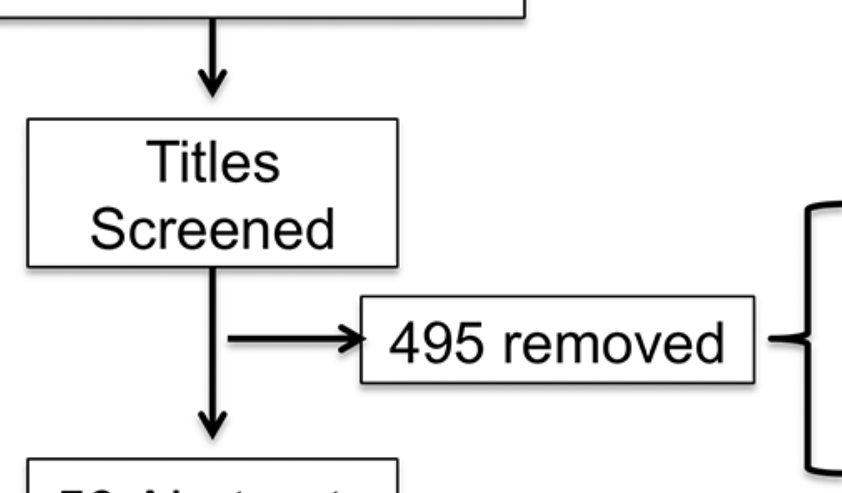

Not specific to depression, $\mathrm{n}=23$

Not specific to HIV, $n=61$

Pediatric study, $\mathrm{n}=48$

Not an intervention, $n=298$

Perinatal depression, $n=50$

Qualitative, $n=9$

50 Abstracts

Screened

26 Full Texts

Screened

for Eligibility

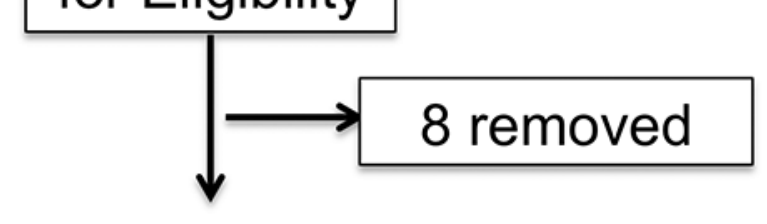

\section{Articles}

Intervention is ART, $n=6$

Not specific to depression, $\mathrm{n}=5$

Not specific to HIV, $n=1$

Pediatric study, $\mathrm{n}=1$

Not an intervention, $n=11$

Perinatal depression, $n=1$

Qualitative, $n=1$

Intervention is ART, $n=2$

Not specific to Africa, $n=1$

Not specific to HIV, $n=1$

Depression not measured, $n=1$

Not an intervention, $\mathrm{n}=4$

Intervention is screening not treatment, $n=1$

Not specific to Africa, $n=1$

Figure 1.

Flow chart depicting the number of reports screened and included in the systematic review. 


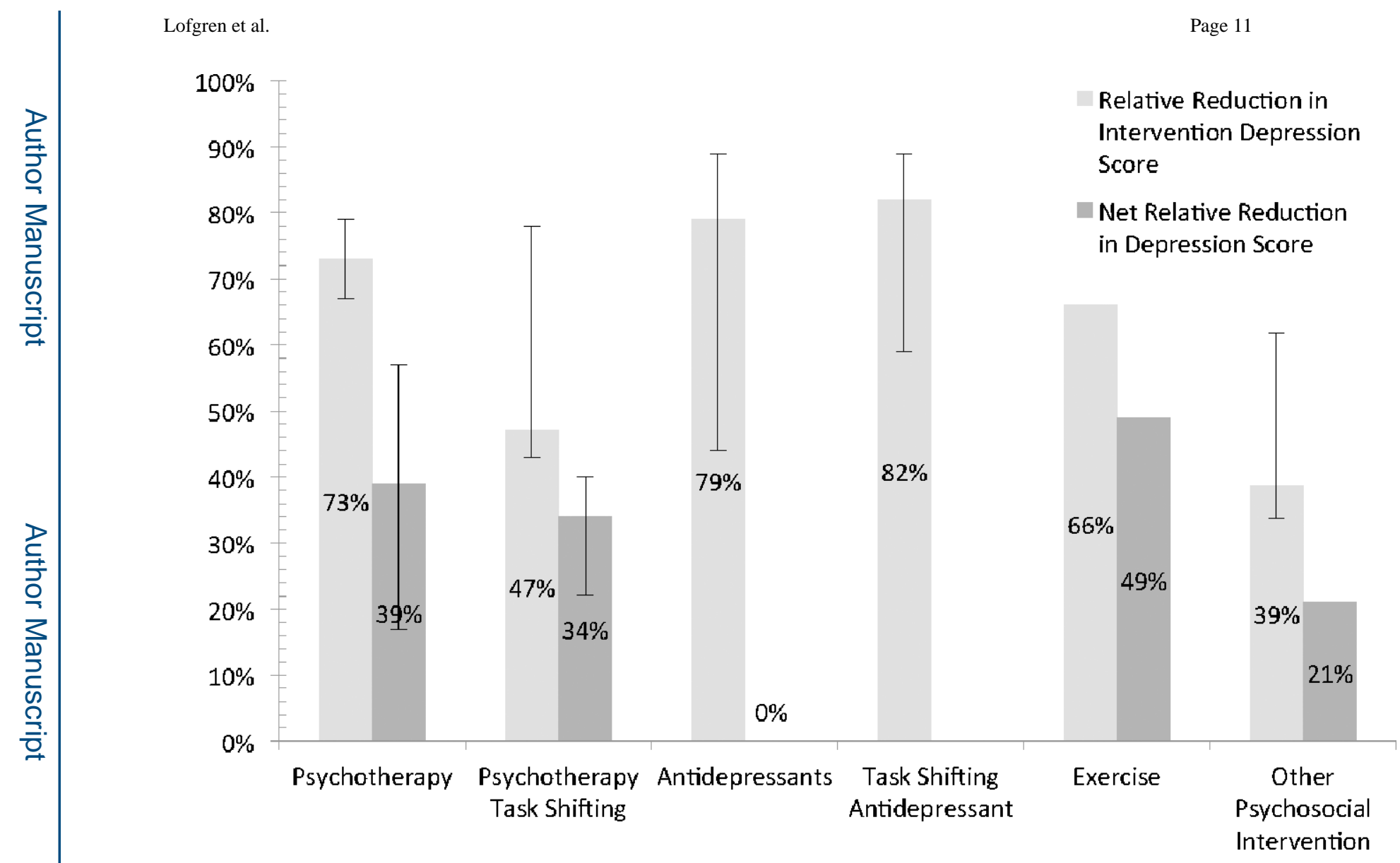

Figure 2.

Weighted Average Effect of Interventions for Depression among HIV-infected Africans Figure 2 presents the weighted average decrease in depression score by intervention type compared with net weighted drop in depression score (of those with control arms). 


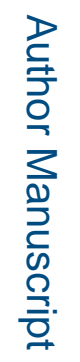

I

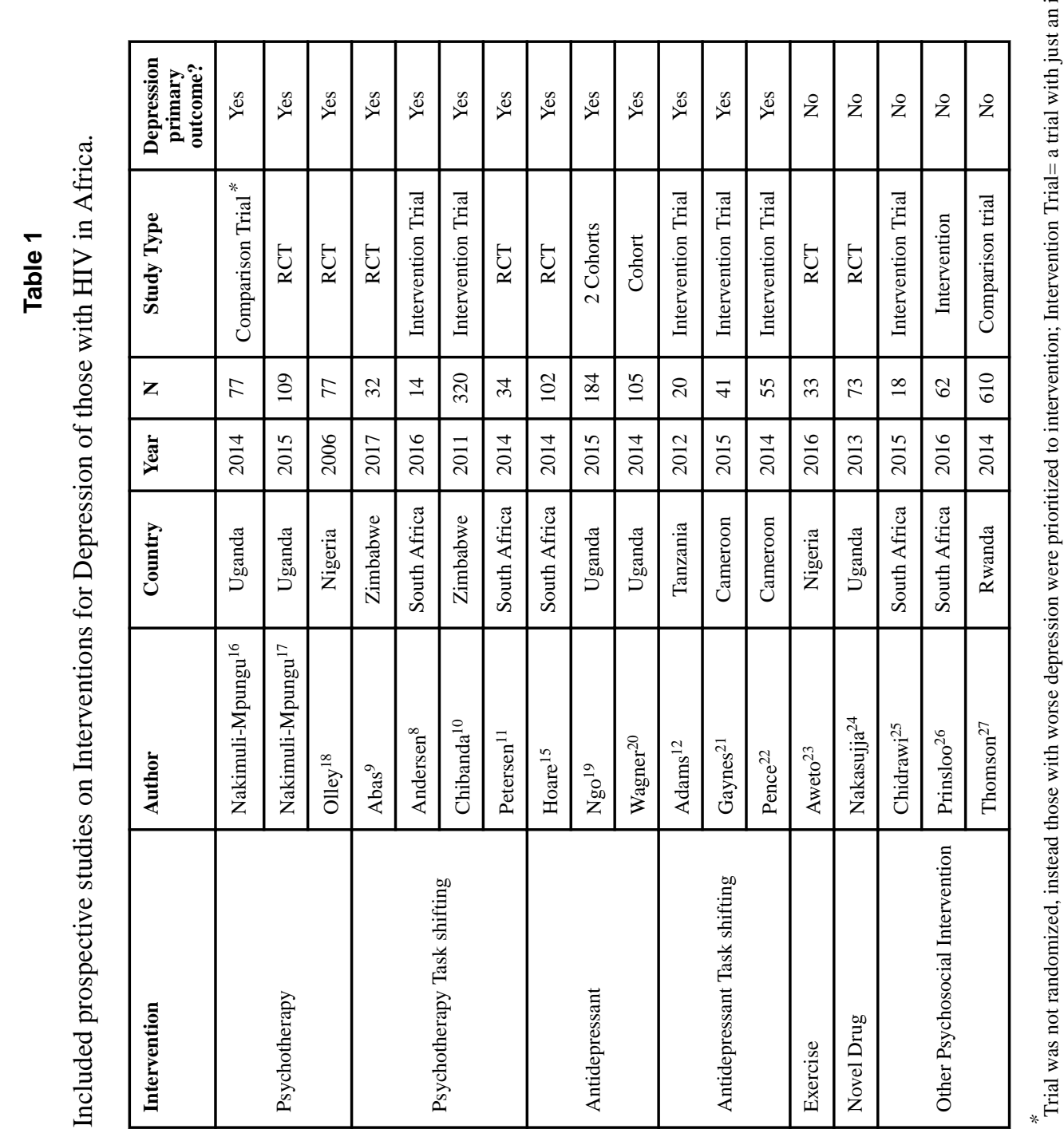

AIDS Behav. Author manuscript; available in PMC 2019 January 01. 


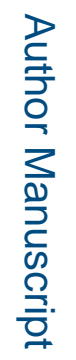

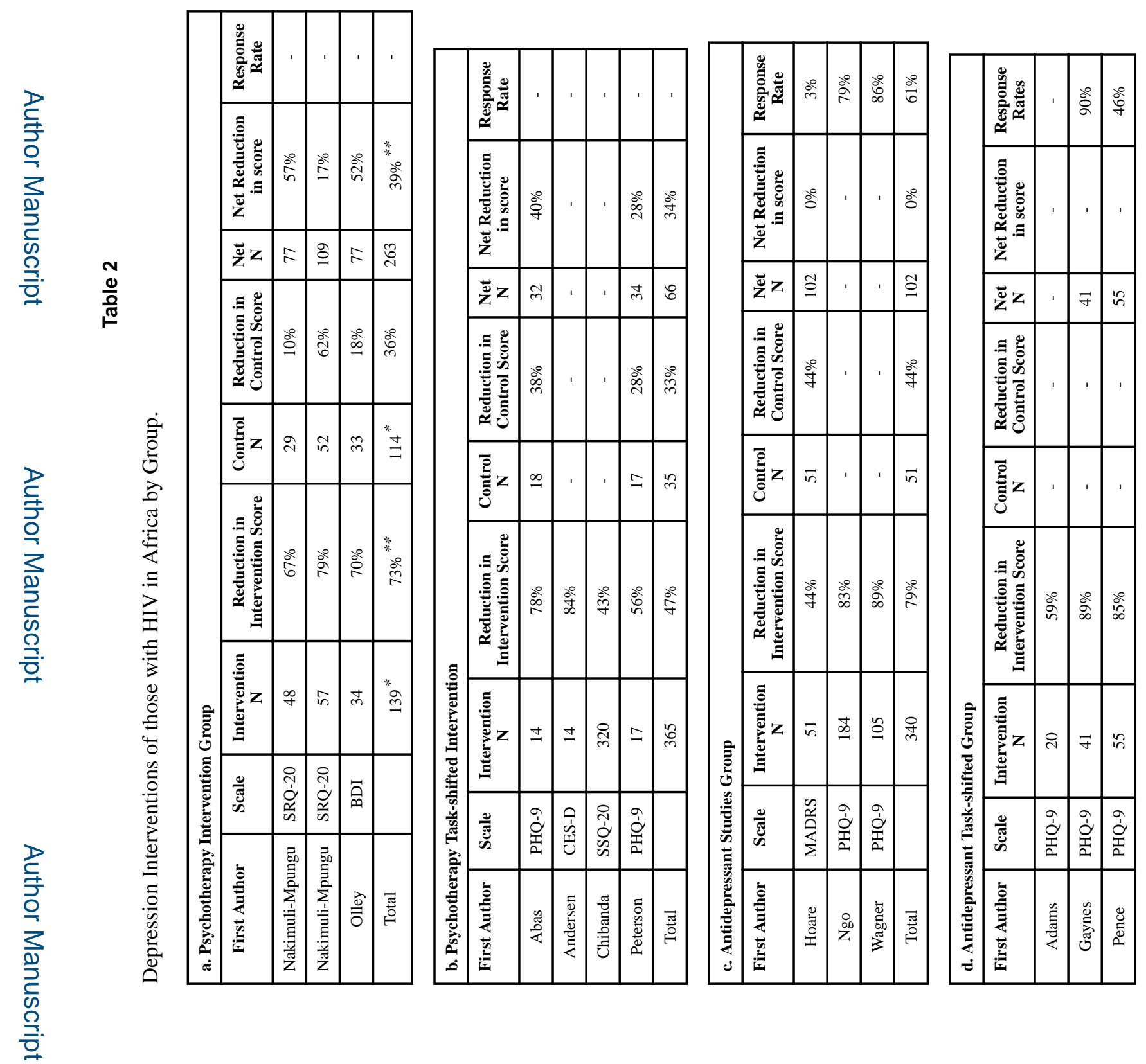

AIDS Behav. Author manuscript; available in PMC 2019 January 01. 


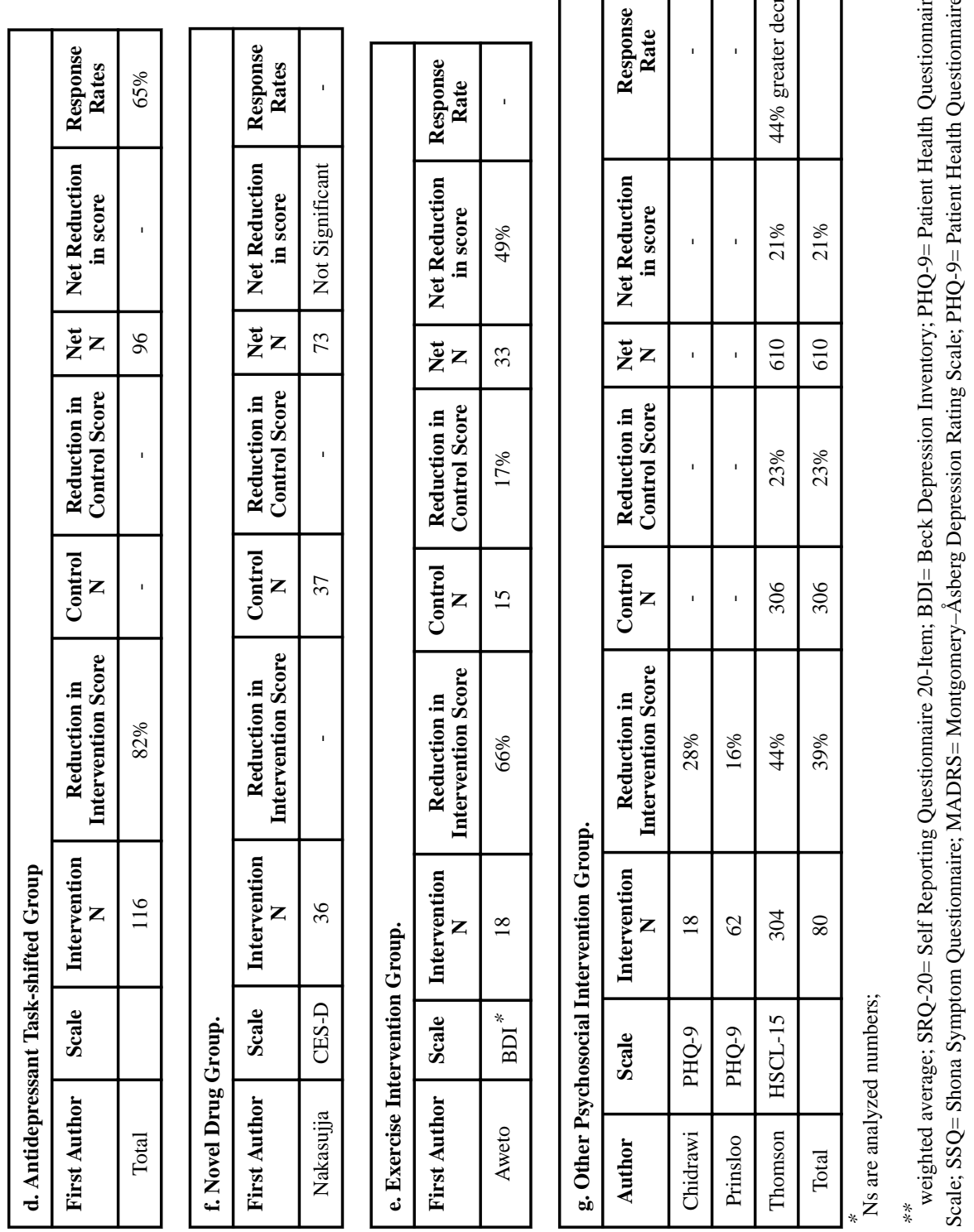

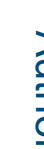

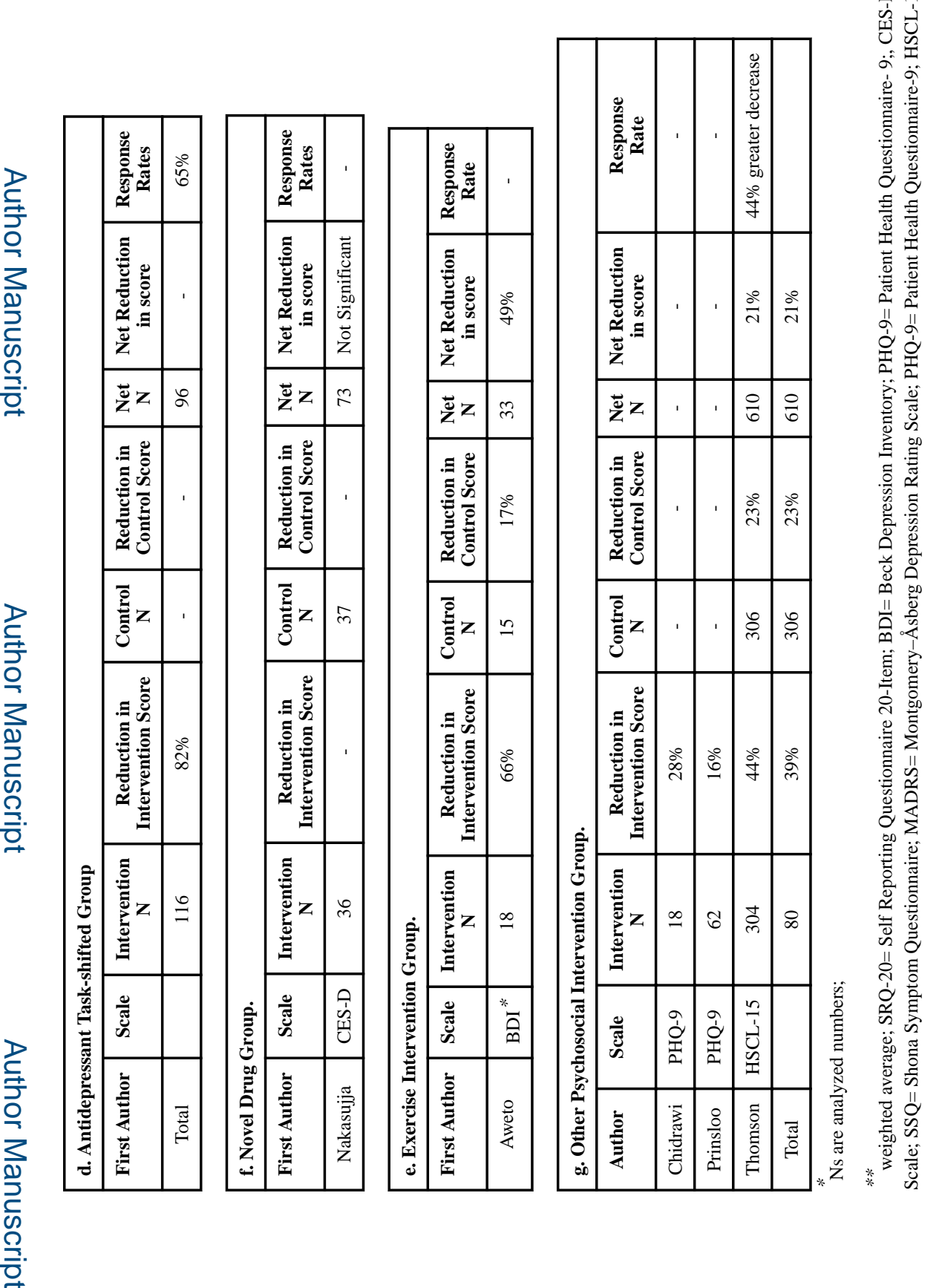

AIDS Behav. Author manuscript; available in PMC 2019 January 01. 